\title{
Systemic Pneumocystis carinii pneumonia prophylaxis with dapsone and pyrimethamine fails to protect against extrapulmonary pneumocystosis
}

\author{
E M Carlin, R J Coker, R D Goldin, J R W Harris
}

\begin{abstract}
Extrapulmonary pneumocystosis is a feature of severe immunosuppression which earlier reports have suggested is limited to patients receiving either no prophylaxis or aerosolised pentamidine. We report a case of disseminated pneumocystosis which developed in an HIV positive homosexual man despite systemic primary Pneumocystis carinii pneumonia prophylaxis.
\end{abstract}

(Genitourin Med 1993;69:130-132)

\section{Introduction}

Pneumocystis carinii pneumonia (PCP) is the commonest severe opportunist infection seen in patients infected with the human immunodeficiency virus (HIV). ${ }^{1}$ This is despite effective prophylaxis which is now recommended for all patients with the acquired immunodeficiency syndrome (AIDS), those with CD4 count $<200 / \mathrm{mm}^{3}$, and those with symptomatic HIV disease. ${ }^{2}$ Patients with AIDS are now surviving longer ${ }^{3}$ and the complications of more severe immunosuppression are being seen. Amongst these is disseminated pneumocystosis. This report is the first, to our knowledge, to show that disseminated pneumocystosis can occur in patients receiving systemic PCP prophylaxis.

\section{Case report}

A 34 year old homosexual was admitted in his final illness with a one month history of a fever, dyspnoea and a cough productive of clear sputum which had failed to respond to amoxycillin. He had been diagnosed seropositive for the human immunodeficiency virus (HIV) four years earlier. The acquired immunodeficiency syndrome (AIDS) was diagnosed when cytomegalovirus retinitis occurred 14 months prior to his final presentation. He was treated with ganciclovir via a portacath. However, 6 months later his retinitis relapsed, reinduction with foscarnet was complicated by renal failure and ganciclovir was reintroduced as maintenance therapy. His renal function improved but he was left with some residual renal insufficiency. Three months prior to admission he had a brief episode of fever, examination was normal but the chest radiograph showed bilateral hilar lymphadenopathy. He declined further investigation.

Primary Pneumocystis carinii pneumonia
(PCP) prophylaxis was started 18 months prior to his last admission, initially as fort $\stackrel{\overrightarrow{\vec{S}}}{\mathrm{D}}$ nightly aerosolised pentamidine $(300 \mathrm{mg}$ via Respirgard II nebuliser) and then, after $11 \stackrel{\overline{\underline{\sigma}}}{\mathrm{\sigma}}$ months, changed to dapsone $(100 \mathrm{mg}$ twices weekly) and pyrimethamine $(25 \mathrm{mg}$ twiceo weekly). He claimed to be compliant with alles medication. He had taken zidovudine for $1 \vec{\circ}$ year 3 years earlier but this had been stoppedbecause of anaemia.

On examination he was pyrexial $\left(40^{\circ} \mathrm{C}\right)$. Bilateral basal crepitations were audible in theo chest and extensive inactive retinitis was pre sent in the right fundus with reduced acuity to hand movements only. Cardiovascular, neurological and abdominal examinations? were normal.

Investigations showed haemoglobin $11.8 \mathrm{~g} / \mathrm{l}$, white cell count $2.7 \times 10^{9} / 1$, neutrophils $1.2 \times 10^{9} / 1$, platelets $69 \times 10^{9} / 1,0$ CD4 count $10 / \mathrm{mm}^{3}$ Serum creatinine $245 \mu \mathrm{mol} / \mathrm{l}$, creatinine clearance $29.5 \mathrm{ml} / \mathrm{min}$, AST $127 \mathrm{U} / 1$, ALT $95 \mathrm{U} / 1$, alkaline phosphatase $290 \mathrm{U} / 1$, bilirubin $6 \mu \mathrm{mol} / 1$. Chest radiography (fig 1) showed bilateral hilar lym $\frac{0}{0}$ phadenopathy, lower zone interstitial infil trates, and small bilateral pleural effusions $\frac{O}{\vec{O}}$ Arterial oxygen saturation on air was $85 \%$. Abdominal CT showed a single prominento

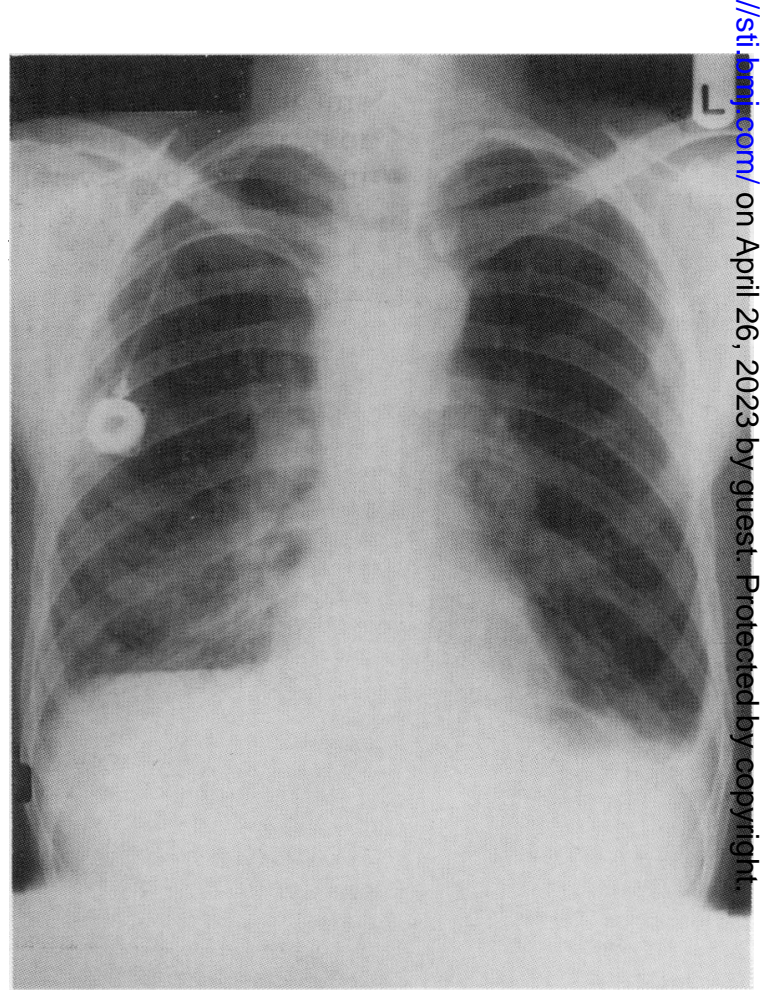

Figure 1 Chest radiograph on admission showing bilateral hilar lymphadenopathy, basal interstitial infiltrates, and small bilateral pleural effusions. 


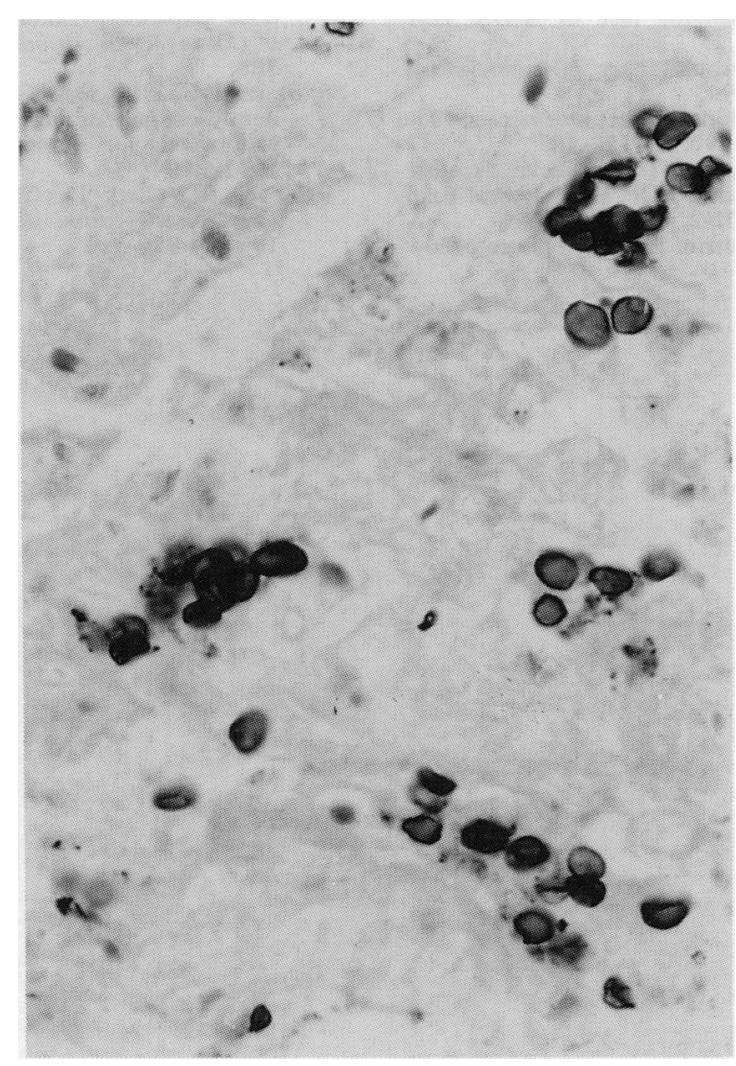

Figure 2 Cyst forms of Pneumocystis carinii seen in the tracheo-bronchial lymph nodes (Grocott's methenamine silver stain). Magnification $\times 1000$ under oil immersion.

para-aortic lymph node. Stool, urine, blood and sputum cultures including mycobacterial culture were negative.

High dose co-trimoxazole (sulphamethoxazole $5.6 \mathrm{~g}$ and trimethoprim $1 \cdot 12 \mathrm{~g} / 24 \mathrm{~h}$ ) and cefotaxime ( $2 \mathrm{~g}$ qds) were commenced intravenously. There was no significant improvement and 2 days later he deteriorated further with the sudden onset of left pleuritic chest pain, increasing hypoxia and dyspnoea. Two days later he developed a rash, sulphonamide allergy was suspected and so co-trimoxazole was discontinued and dapsone $(100 \mathrm{mg}$ od) orally and trimethoprim (15 mg/kg/24hrs) intravenously were substituted. In addition intravenous flucloxacillin ( $2 \mathrm{~g}$ qds), ciprofloxacin (200 mg bd), and oral rifampicin $(600 \mathrm{mg} \mathrm{od})$, and pyrazinamide (1 $\mathrm{g} \mathrm{od})$ were added.

He deteriorated further with the spontaneous development of bilateral pneumothoraces. Neither CPAP nor mechanical ventilation were used. Bilateral intercostal drains were inserted but there was no improvement and he died 7 days later.

Post mortem examination revealed diffuse consolidation in both lungs and discrete haemorrhagic, necrotic lesions in the right lung unrelated to lymph nodes. The lungs showed the histological features of the proliferative (organising) phase of diffuse alveolar damage (adult respiratory distress syndrome) with a patchy chronic inflammatory infiltrate, interstitial oedema and prominent type II pneumocytes. Scattered foci of calcification of the alveolar exudate were present. In addition there were larger areas of confluent necrosis and pneumocysts were seen on staining with Grocott's methenamine silver stain. The tracheo-bronchial, coeliac axis and para-aortic lymph nodes were enlarged and showed haemorrhage and necrosis. Histological examination of lymph nodes from all of these sites showed an eosinophilic granular exudate in which Pneumocystis carinii cysts could be demonstrated on staining with Grocott's methenamine silver stain (fig 2 ).

\section{Discussion}

Extrapulmonary pneumocystosis occurs in up to $2.5 \%$ of AIDS patients, ${ }^{4}$ and several studies have shown that the diagnosis is often made post mortem. ${ }^{5}$ Earlier reports have suggested that systemic PCP prophylaxis confers protection.

In this case it may be that subclinical PCP was present prior to the introduction of systemic prophylaxis 7 months before his death. However, the lack of symptoms prior to this period suggest this is unlikely, particularly as primary prophylaxis with aerosolised pentamidine is effective. However, the effect of pentamidine in the severely immunocompromised with low CD4 counts has been questioned. Earlier reports have suggested that dapsone $100 \mathrm{mg}$ and pyrimethamine $25 \mathrm{mg}$ twice weekly are also effective in preventing PCP $^{6}$ but recently at this dose dapsone and pyrimethamine have been shown to be less efficacious in preventing PCP than pentamidine or co-trimoxazole. ${ }^{7}$ Our experience would agree with this and we are currently studying data from our own unit.

Compliance in patients is a problem which is difficult to evaluate long term patient acceptability is important and is known to be higher with pentamidine than co-trimoxazole. $^{8}$

Extrapulmonary pneumocystosis is a problem among long-term survivors with AIDS. As the survival of patients with AIDS increases the frequency of disseminated pneumocystosis can be expected to increase. Both clinicians and pathologists are becoming increasingly aware of disseminated pneumocystosis. As a result of earlier cases at both St. Mary's and Middlesex Hospitals ${ }^{9}$ we routinely look for evidence of pneumocysts in all clinical material from patients with HIV.

In conclusion, systemic prophylaxis with dapsone and pyrimethamine, a regimen now known to be less effective than co-trimoxazole in a patient with uncertain compliance, does not confer protection against the development of extrapulmonary PCP. We suggest that clinicians should be alert to this condition as early intervention may improve prognosis.

1 Miller RF, Mitchell DM. Pneumocystis carinii pneumonia. Thorax 1992;47:305-14

2 Girard PM, Pocidalo JJ, Murray JF. Primary prophylaxis against common infectious diseases in persons with human immunodeficiency virus infection. $A m \quad R e v$ Respir Dis 1991;143:447-50

3 Peters BS, Beck EJ, Coleman DG, et al. Changing disease patterns in patients with AIDS in a referral centre in the 
United Kingdom: the changing face of AIDS. $B M 9$ 1991;302:203-7.

4 Coker RJ, Peters BS. Extrapulmonary pneumocystosis. Ann Intern Med 1991;115:499.

5 Raviglione MC. Extrapulmonary pneumocystosis: The first 50 cases. Rev Inf Dis 1990;12:1127-38.

6 Clotet B, Sirera G, Romeu J, et al. Twice-weekly dapsonepyrimethamine for preventing PCP and cerebral toxoplasmosis. AIDS 1991;5:601-2

7 Antinori A, Murri R, Tamburrini E, et al. Failure of low- dose dapsone-pyrimethamine in primary prophylaxis of Pneumocystis carinii pneumonia. Lancet 1992;340: of Pneumocystis carinii pneumonia. Lancet 1992;340:
788 . 8 Smith DE, Hills DA, Gazzard BG. Patient tolerance of nebulised pentamidine or co-trimoxazole as secondary
prophylaxis for Pneumocystis carinii pneumonia. AIDS

9 Coker RJ, Clark D, Claydon EL, et al. Disseminated Pneumocystis carinii infection in AIDS. $\mathcal{f}$ Clin Pathol 1991;44:820-3. 